\title{
Robust Design Optimization via Failure Domain Bounding
}

\author{
Luis G. Crespo* \\ National Institute of Aerospace. \\ Sean P. Kenny ${ }^{\dagger}$ and Daniel P. Giesy ${ }^{\dagger}$ \\ Dynamic Systems and Control Branch, NASA Langley Research Center.
}

This paper extends and applies the strategies recently developed by the authors for handling constraints under uncertainty to robust design optimization. For the scope of this paper, robust optimization is a methodology aimed at problems for which some parameters are uncertain and are only known to belong to some uncertainty set. This set can be described by either a deterministic or a probabilistic model. In the methodology developed herein, optimization-based strategies are used to bound the constraint violation region using hyper-spheres and hyper-rectangles. By comparing the resulting bounding sets with any given uncertainty model, it can be determined whether the constraints are satisfied for all members of the uncertainty model (i.e., constraints are feasible) or not (i.e., constraints are infeasible). If constraints are infeasible and a probabilistic uncertainty model is available, upper bounds to the probability of constraint violation can be efficiently calculated. The tools developed enable approximating not only the set of designs that make the constraints feasible but also, when required, the set of designs for which the probability of constraint violation is below a prescribed admissible value. When constraint feasibility is possible, several design criteria can be used to shape the uncertainty model of performance metrics of interest. Worst-case, least-second-moment, and reliability-based design criteria are considered herein. Since the problem formulation is generic and the tools derived only require standard optimization algorithms for their implementation, these strategies are easily applicable to a broad range of engineering problems.

${ }^{*}$ Staff Scientist, 100 Exploration Way, Hampton VA 23666. Member AIAA.

${ }^{\dagger}$ Aerospace Technologist, Mail Stop 308, NASA LaRC, Hampton VA, 23681. 
Keywords: design under uncertainty, robust optimization, critical parameter value, parametric safety margin, reliability indices, failure probability bounds.

\section{List of Acronyms}

$\begin{array}{ll}\text { CPV } & \text { Critical Parameter Value } \\ \text { CSR } & \text { Critical Similitude Ratio } \\ \text { FDS } & \text { Feasible Design Space } \\ \text { FORM } & \text { First Order Reliability Method } \\ \text { MCS } & \text { Monte Carlo Sampling } \\ \text { PSM } & \text { Parametric Safety Margin } \\ \text { RDS } & \text { Robust Design Space } \\ \text { RI } & \text { Reliability Index }\end{array}$

\section{Introduction}

Design under uncertainty arises in numerous disciplines including engineering, economics, finance, and management. Achieving balance between robustness and performance is one of the fundamental challenges faced by scientists and engineers. Trade-offs should be made to meet acceptable levels of performance with adequate robustness to uncertainty.

The concern in this paper is the analysis and design of a system described by a parametric mathematical model. The parameters which specify the system are grouped into two categories: uncertain parameters, which are denoted by the vector $\boldsymbol{p}$, and design parameters, which are denoted by the vector $\boldsymbol{d}$. For example, one component of $\boldsymbol{d}$ could be the designer's choice for the thickness of a structural member while one component of $\boldsymbol{p}$ could represent the manufacturing error in that same thickness or uncertainty in some material property. For purposes of using the design techniques presented here, the necessary information about the system being designed is abstracted in constraint functions and a performance metric. These are functions of $\boldsymbol{p}$ and $\boldsymbol{d}$. The constraint functions are scaled so that they take on positive values at parameter values representing unacceptable systems. The design process attempts to find a design which is acceptable over the entire range of uncertainty and which optimizes (in a sense to be made precise subsequently) the performance metric. The uncertainty in $\boldsymbol{p}$ is modeled by specifying a set, the so-called uncertainty set $\Delta_{\boldsymbol{p}}$. While the actual value of $\boldsymbol{p}$ is uncertain, it is assumed that $\boldsymbol{p} \in \Delta_{\boldsymbol{p}}$. One way of specifying $\Delta_{\boldsymbol{p}}$ will be termed "deterministic". For example, the uncertain manufacturing error mentioned earlier could be 
modeled as an interval of real numbers based on known manufacturing tolerances. Another way of specifying uncertainty is to model $\boldsymbol{p}$ as a random vector. Then $\Delta_{\boldsymbol{p}}$ could be taken as the Support Set of its joint Probability Density Function (PDF), so $P\left[\boldsymbol{p} \notin \Delta_{\boldsymbol{p}}\right]=0$ (if $f_{\boldsymbol{p}}(\boldsymbol{p})$ is the joint PDF of $\boldsymbol{p}$, take $\left.\Delta_{\boldsymbol{p}}=\left\{\boldsymbol{p}: f_{\boldsymbol{p}}(\boldsymbol{p}) \neq 0\right\}\right)$. Hereafter, the terms "uncertainty set" and "support set" will be considered equivalent. In principle, the uncertainty set can be unbounded. In practical applications, the choice of $\Delta_{p}$ is usually made by a discipline expert. However, the theory presented subsequently acknowledges that such a choice may be fairly arbitrary, and addresses the need for quantifying the level of tolerance of the system to uncertainty in $\boldsymbol{p}$ when a particular $\boldsymbol{d}$ is chosen. Any member of the uncertainty set is called a Realization. For all the variables that depend on $\boldsymbol{p}$, such as performance metrics and constraints, there is a corresponding uncertainty model. The uncertainty model of these variables is fully prescribed by the uncertainty model of $\boldsymbol{p}$ and by the equation that relates the dependent variable with $\boldsymbol{p}$.

Design requirements for the system are prescribed by means of a vector of constraint functions, $\boldsymbol{g}(\boldsymbol{p}, \boldsymbol{d}) \leq \mathbf{0}$, which in general depend on both the uncertain and design parameters. The system is deemed acceptable if the constraints are satisfied for all possible realizations of the uncertain parameter. For each value of the design parameter vector, these functions partition the uncertain parameter space into two sets, a Failure Domain, where the design requirements are not satisfied because at least one of the constraints is violated, and its complement set, the non-failure domain where no constraints are violated.

For a fixed design point $\boldsymbol{d}$ and a given uncertainty model of $\boldsymbol{p}$, the constraints $\boldsymbol{g}(\boldsymbol{p}, \boldsymbol{d}) \leq \mathbf{0}$ are said to be Feasible, if they are satisfied for all possible realizations of the uncertain parameter. Otherwise, the constraints are Infeasible. Robust optimization strategies can be classified according to the ability and/or desire to make constraints feasible. Constraint feasibility is usually imposed on critical design requirements. Previous strategies ${ }^{1-5}$ to solve the resulting semi-infinite optimization problem require nested searches to identify the WorstCase Parameter Realization. ${ }^{1}$ In previous work, ${ }^{6,7}$ we present constraint feasibility tests based on the deformation of sets in $\boldsymbol{p}$-space and the calculation of Critical Parameter Values (CPVs) and Parametric Safety Margins (PSMs). These methods are optimization based, and the results are reliable so long as global optima have been obtained.

By necessity or desire, one may want to let some parameter realizations violate the constraints. This situation may result from slack design requirements, overly large uncertainty sets and limiting design architectures. Deterministic ${ }^{5}$ and probabilistic ${ }^{8-11}$ approaches, 
including chance-constrained programming, ${ }^{12}$ sampling-based techniques, reliability-based methods, ${ }^{13}$ and penalty-based optimization, ${ }^{5,12}$ are available. The developments in prior work $^{14,15}$ use probabilistic uncertainty models and focus on strategies for limiting the probability that $\boldsymbol{p}$ will fall into the Failure Domain. Hereafter, the probability that the uncertain parameter falls in the Failure Domain will be referred to as the failure probability. In these studies, ${ }^{8-11}$ closed-form expressions for upper bounds to the failure probability are derived along with a hybrid method for the approximation of its actual value. Analogous to constraint feasibility tests in the previous work, ${ }^{6,7}$ this optimization-based methodology is based on the deformation of sets, now in the standard normal space, and on the calculation of CPVs and Reliability Indices (RIs).

In this paper, the deformations mentioned above are expansions or contractions that preserve proportionality and orientation. These are called homothetic deformations. The methods developed in the prior work ${ }^{6,7,14,15}$ are based on the homothetic deformation of hyper-spherical or hyper-rectangular sets until the deformed set is of maximal size without encroaching on the Failure Domain. For these two geometries, we cast the problem of finding the maximal homothetic deformation in terms of an optimization problem for which standard nonlinear constrained optimization algorithms are applicable. Because the Failure Domain depends upon the design variable, the larger the maximal homothetic deformation, the better the robustness characteristics of the corresponding design. Therefore, one can then search for a design which admits the largest maximal homothetic deformation. Design optimization methods based on this idea are presented herein.

When there are designs for which the constraints are feasible, we can attempt to select one of these designs which optimizes the value of a performance function $y(\boldsymbol{p}, \boldsymbol{d})$. However, since $\boldsymbol{p}$ can be any point in the uncertainty set, $y$ can take on many values for each feasible design; these values comprise the uncertainty model of $y$ at the design point. Several design criteria, in the form of functionals $J$ which operate on these uncertainty models, will be introduced to provide a single value of merit from this collection of possible performance function values. The design criteria considered in this paper are worst-case, least-secondmoment and reliability-based. The robust constrained optimization problem of interest will lead to designs for which the uncertainty model of $y$ is optimal while the constraints remain feasible.

The content of this paper is organized as follows. The problem formulation and basic mathematical background is described in Section II. An overview on how to study and 
integrate inequality constraints into robust optimization problems is presented in Section III. The optimal design criteria mentioned above are then introduced in Section IV and exemplified with a low-dimensional example in Section V. Finally, Section VI presents conclusions.

\section{Problem Formulation}

A design procedure using a conventional constrained optimization problem with no uncertainty wherein it is assumed that the parameter $\boldsymbol{p}$ is equal to a known value $\overline{\boldsymbol{p}}$ is given by the following problem statement:

\section{Problem 1 (Deterministic Optimization Problem).}

$$
\min _{\boldsymbol{d}}\{y(\overline{\boldsymbol{p}}, \boldsymbol{d}): \boldsymbol{g}(\overline{\boldsymbol{p}}, \boldsymbol{d}) \leq \mathbf{0}\}
$$

Here, $y$ is a performance metric of interest and $\boldsymbol{g}$ is a set of constraint functions for which the inequalities $\boldsymbol{g} \leq \mathbf{0}$ correspond to a set of requirements for which violations are unacceptable. The cases in which some violations are acceptable or even unavoidable are considered later (Section IV.A.3 and Section IV.B, respectively). Now we consider the case in which $\boldsymbol{p}$ is uncertain and we have modeled the uncertainty with an uncertainty set $\Delta_{\boldsymbol{p}}$. For a given design point, the propagation of $\Delta_{p}$ through $y$ leads to the uncertainty set $\Delta_{y}(\boldsymbol{d}) \triangleq y\left(\Delta_{\boldsymbol{p}}, \boldsymbol{d}\right)$. In this case, the counterpart to Problem 1 is given in the following problem statement:

\section{Problem 2 (Robust Optimization Problem).}

$$
\min _{\boldsymbol{d}}\left\{J\left(\Delta_{y}(\boldsymbol{d})\right): \boldsymbol{g}(\boldsymbol{p}, \boldsymbol{d}) \leq \mathbf{0} \text { for all } \boldsymbol{p} \in \Delta_{\boldsymbol{p}}\right\}
$$

Here, $J(\cdot)$ assigns a scalar to each uncertainty model $\Delta_{y}(\boldsymbol{d})$. For example, to minimize the worst case value of $y, J\left(\Delta_{y}\right)$ would be chosen to be $\max \left(\Delta_{y}\right)$. Observe that Problem 1 is the special case of Problem 2 with $\Delta_{p}=\{\overline{\boldsymbol{p}}\}$ and $J$ being (essentially) the identity function. If $\boldsymbol{p}$ is modeled as a random vector and $\Delta_{\boldsymbol{p}}$ is the support of its PDF, any design $\boldsymbol{d}$ which satisfies the constraint $\boldsymbol{g}(\boldsymbol{p}, \boldsymbol{d}) \leq \mathbf{0}$ for all $\boldsymbol{p} \in \Delta_{\boldsymbol{p}}$ in Problem 2 has zero probability of violating the constraints.

The robust optimization problems above deal with the search for design points that shape 
the uncertainty model $\Delta_{y}$ of $y$, directly in the deterministic case and indirectly by shaping $f_{y}(y)$ in the probabilistic one, according to the optimality criterion prescribed by $J$, while limiting the search to designs $\boldsymbol{d}$ for which no vector in the set $\boldsymbol{g}\left(\Delta_{\boldsymbol{p}}, \boldsymbol{d}\right)$ has any positive components. For instance, in a structural problem $\boldsymbol{p}$ could contain material properties, $\boldsymbol{d}$ might parameterize the geometry of the structure, and $\boldsymbol{g} \leq \mathbf{0}$ might be a requirement on the maximum allowable stress. If $y$ is the weight of the structure, whose dependence on $\boldsymbol{p}$ makes it uncertain as well, $J(y)$ might be the worst-case weight.

\section{Robustness Analysis}

The tools introduced in this section enable the quantification of the robustness of the system and provide the means to efficiently implement a constraint feasibility test in the form of a constraint function for an optimization problem and to calculate upper bounds of failure probability.

In an earlier paper, ${ }^{7}$ we introduced the notions of a Reference Set $\Omega_{\boldsymbol{p}}$ in $\boldsymbol{p}$-space and a distinguished realization of the uncertain parameter, $\overline{\boldsymbol{p}} \in \Omega_{\boldsymbol{p}}$, called the Designated Point, which can be regarded as the nominal value of the uncertain parameter. For clarity sake, the presentation of the material will concentrate on the case where the Designated Point is feasible; i.e., $\boldsymbol{g}(\overline{\boldsymbol{p}}, \boldsymbol{d}) \leq \mathbf{0}$. By consideration of maximal homothetic deformations of the Reference Set which did not intersect the Failure Domain (which is a function of the chosen design), a measure of the robustness of that design to parameter uncertainty was obtained. One reasonable choice for the Reference Set is a hyper-sphere. The hyper-sphere of radius $R$ centered at $\overline{\boldsymbol{p}}$, denoted as $\mathcal{S}(\overline{\boldsymbol{p}}, R)$, is defined by

$$
\mathcal{S}(\overline{\boldsymbol{p}}, R)=\{\boldsymbol{p}:\|\overline{\boldsymbol{p}}-\boldsymbol{p}\| \leq R\}
$$

where $\|\cdot\|$ denotes the $\ell_{2}$ norm in $\mathbb{R}^{\operatorname{dim}(\boldsymbol{p})}$ space. Another reasonable choice might be to confine each component of the Reference Set to a bounded interval. This leads to a hyper-rectangular set. If $\boldsymbol{m}$ is the vector of half-lengths of the sides of the rectangle, the hyper-rectangle $\mathcal{R}(\overline{\boldsymbol{p}}, \boldsymbol{m})$ is defined by

$$
\mathcal{R}(\overline{\boldsymbol{p}}, \boldsymbol{m})=\left\{\boldsymbol{p}: \boldsymbol{p}_{i} \in\left[\overline{\boldsymbol{p}}_{i}-\boldsymbol{m}_{i}, \overline{\boldsymbol{p}}_{i}+\boldsymbol{m}_{i}\right], 1 \leq i \leq \operatorname{dim}(\boldsymbol{p})\right\}
$$

Hyper-spherical sets consider parameters with similar levels of uncertainty. They could also 
be used for parameters with dissimilar levels of uncertainty if some scaling is used. However, whether scaling is used or not, a degree of dependence is introduced. For instance, the range of variability of one parameter depends on the values taken on by the others. Rectangular sets permit the consideration of dissimilar levels of uncertainty without the need for scaling and introduce no such dependence. A design point is deemed to be in the Feasible Design Space (FDS) if it satisfies all the constraints when the uncertain parameter assumes the value of the Designated Point. A design is a Robust Design if it is a feasible design for every choice of the uncertain parameter in the uncertainty set. The Robust Design Space (RDS) is the set of all Robust Designs. Note that if the RDS exists, it is a subset of the FDS. This is so since the implied constraint set $\{\overline{\boldsymbol{p}}\}$ for $\boldsymbol{p}$ in Problem 1 is a subset of the constraint set in Problem 2. For a fixed design point $\boldsymbol{d}$, the Failure Region, denoted as $\mathcal{F}(\boldsymbol{d}, \boldsymbol{g})$, is composed of the parameters that do not satisfy all the constraints, and is given by the union of the Failure Domains for each individual constraint $\mathcal{F}_{i}(\boldsymbol{d}, \boldsymbol{g})=\left\{\boldsymbol{p}: \boldsymbol{g}_{i}(\boldsymbol{p}, \boldsymbol{d})>0\right\}$, where $i=1, \ldots \operatorname{dim}(\boldsymbol{g})$.

One of the tasks of interest is to assign a measure of robustness to a design point based on measuring how much the Reference Set can be deformed before intersecting the Failure Domain. Mathematical structure is imposed on this process by limiting attention to homothetic deformations. A Homothetic Deformation by a factor of $\alpha$ can be viewed in the following way. Imagine standing at the Designated Point and looking at any other point of $\Omega_{\boldsymbol{p}}$. Denote the distance from the Designated Point to the other point by $\delta$. The corresponding point of the Homothetic Deformation of $\Omega_{\boldsymbol{p}}$ with similitude ratio $\alpha$ is attained by looking in the same direction and placing the point at a distance of $\alpha \delta$ from the Designated Point. A mathematical expression for the corresponding deformed set is $\left\{\boldsymbol{q}: \boldsymbol{q}=\overline{\boldsymbol{p}}+\alpha(\boldsymbol{p}-\overline{\boldsymbol{p}}), \boldsymbol{p} \in \Omega_{\boldsymbol{p}}\right\}$. While expansions are accomplished when $\alpha>1$, contractions result when $\alpha<1$. Hereafter, deformations must be interpreted as homothetic expansions or contractions.

For purposes of this paper, two uncertainty models will be called Proportional if one of the two sets can be formed from the other by homothetic deformation by some positive factor about the common Designated Point. Such a factor is called the Similitude Ratio and is denoted by $\alpha$. For instance, the hyper-rectangles $\mathcal{R}(\overline{\boldsymbol{p}}, \boldsymbol{m})$ and $\mathcal{R}(\overline{\boldsymbol{p}}, \alpha \boldsymbol{m})$ are proportional sets.

The notions of Critical Parameter Value and Parametric Safety Margin are now introduced. The reader should keep in mind that these are functions of the design point, since they depend on the Failure Domain which, itself, depends on the design point. Intuitively, 
one imagines that a set proportional to the Reference Set is being expanded homotheticly with respect to its Designated Point, until its boundary just touches the boundary of the Failure Domain. The point(s) where the expanding set touches the Failure Domain is (are) the CPV(s). The Critical Similitude Ratio (CSR), denoted as $\tilde{\alpha}$, is the similitude ratio of that expansion, and the PSM, which will be defined subsequently for the cases that the Reference Set is a hyper-sphere or a hyper-rectangle, is a dimensional metric that quantifies the size of the corresponding deformed set. Both the CSR and the PSM are measures of robustness of a design to parameter uncertainty. The larger these metrics are, the larger the variation of the uncertain parameter from the Designated Point that can occur before encountering a constraint violation. Therefore, both are metrics which quantify the degree of robustness of $\boldsymbol{d}$ to uncertainty in $\boldsymbol{p}$.

The CSR is non-dimensional, but depends on both the shape and the size of the Reference Set. The PSM has the same units as the uncertain parameters, and depends on the shape, but not the size of the Reference Set. If the PSM assumes the value of zero, there is no robustness since at least one of the constraints is active for $\overline{\boldsymbol{p}}$. The convention is that designs within the FDS assume non-negative PSM values, otherwise they are negative.

In the case that the Designated Point $\overline{\boldsymbol{p}}$ satisfies the constraints $(\boldsymbol{g}(\overline{\boldsymbol{p}}, \boldsymbol{d}) \leq \mathbf{0})$ for a given design point $\boldsymbol{d}$, the Spherical PSM, denoted as $\rho_{S}$, is the distance from $\overline{\boldsymbol{p}}$ to a CPV $\tilde{\boldsymbol{p}}$, which is a point on the boundary of the Failure Domain which is closest to the Designated Point. By viewing this as homotheticly deforming the hyper-spherical Reference Set $\Omega_{\boldsymbol{p}}=\mathcal{S}(\overline{\boldsymbol{p}}, R)$ until it touches the constraint violation region, finding $\tilde{\boldsymbol{p}}$ can be cast in terms of an optimization:

$$
\tilde{\boldsymbol{p}}=\tilde{\boldsymbol{p}}^{(i)}
$$

where

$$
i=\underset{1 \leq j \leq \operatorname{dim}(\boldsymbol{g})}{\operatorname{argmin}}\left\{\left\|\tilde{\boldsymbol{p}}^{(j)}-\overline{\boldsymbol{p}}\right\|\right\}
$$

and

$$
\tilde{\boldsymbol{p}}^{(j)}=\underset{\boldsymbol{p}}{\operatorname{argmin}}\left\{\|\boldsymbol{p}-\overline{\boldsymbol{p}}\|: \boldsymbol{g}_{j}(\boldsymbol{p}, \boldsymbol{d}) \geq 0\right\}
$$

(Recall that the mathematical operator "min" returns the minimum value of a function or set, while "argmin" returns the value of a variable at which that minimum occurs.)

In the event that some constraint is violated at $\overline{\boldsymbol{p}}, \mathrm{CPVs}$ are redefined as points in or on the boundary of the constraint satisfaction set which are closest to the Designated Point. 
Such a CPV can be calculated using the previous procedure by reversing the inequality in the optimization given in (3). The fact that, for this design, the Designated Point is in the failure domain is signaled by defining the Spherical PSM to be a negative quantity. The Spherical PSM is now defined as:

$$
\rho_{S} \triangleq \begin{cases}\|\tilde{\boldsymbol{p}}-\overline{\boldsymbol{p}}\| & \text { for } \boldsymbol{g}(\overline{\boldsymbol{p}}, \boldsymbol{d}) \leq \mathbf{0} \\ -\|\tilde{\boldsymbol{p}}-\overline{\boldsymbol{p}}\| & \text { otherwise }\end{cases}
$$

In contrast, in the case that the Designated Point satisfies the constraints at a given design point, the homothetic deformation of the hyper-rectangular Reference Set $\Omega_{\boldsymbol{p}}=\mathcal{R}(\overline{\boldsymbol{p}}, \boldsymbol{m})$ leads to the CPV and CSR given by

$$
\tilde{\boldsymbol{p}}=\tilde{\boldsymbol{p}}^{(i)} \quad \text { and } \quad \tilde{\alpha}=\tilde{\alpha}^{(i)},
$$

where

$$
i=\underset{1 \leq j \leq \operatorname{dim}(\boldsymbol{g})}{\operatorname{argmin}}\left\{\left\|\tilde{\boldsymbol{p}}^{(j)}-\overline{\boldsymbol{p}}\right\|_{\boldsymbol{m}}^{\infty}\right\}
$$

and

$$
\left\langle\tilde{\boldsymbol{p}}^{(j)}, \tilde{\alpha}^{(j)}\right\rangle=\underset{\boldsymbol{p}, \alpha}{\operatorname{argmin}}\left\{\alpha: \boldsymbol{g}_{j}(\boldsymbol{p}, \boldsymbol{d}) \geq 0,\left|\boldsymbol{p}_{k}-\overline{\boldsymbol{p}}_{k}\right| \leq \alpha \boldsymbol{m}_{k}, 1 \leq k \leq \operatorname{dim}(\boldsymbol{p})\right\},
$$

and where the $\boldsymbol{m}$-scaled infinity norm is defined as $\|\boldsymbol{x}\|_{\boldsymbol{m}}^{\infty}=\sup _{i}\left\{\left|\boldsymbol{x}_{i}\right| / \boldsymbol{m}_{i}\right\}$. As in the Spherical case, if the Designated Point does not satisfy the constraints, the CPV and CSR are calculated by reversing the first inequality in (7). The corresponding Rectangular PSM, denoted as $\rho_{R}$, is defined to be:

$$
\rho_{R} \triangleq \begin{cases}\tilde{\alpha}\|\boldsymbol{m}\| & \text { for } \boldsymbol{g}(\overline{\boldsymbol{p}}, \boldsymbol{d}) \leq \mathbf{0} \\ -\tilde{\alpha}\|\boldsymbol{m}\| & \text { otherwise }\end{cases}
$$

Details on the derivation of these expressions are available in an earlier paper. ${ }^{7}$ Figure 1 shows a sketch with relevant metrics for a rectangular Reference Set.

For a given design point and Reference Set, the set proportional to the Reference Set that has the CPV(s) on its boundary is called the Maximal Feasible Homothet and it is denoted as $\mathcal{M}_{\boldsymbol{p}}$ or, if the dependence on $\boldsymbol{d}$ is important, $\mathcal{M}_{\boldsymbol{p}}(\boldsymbol{d})$. The title of this paper refers to the fact that when the constraints are satisfied at the Designated Point, the complement set 


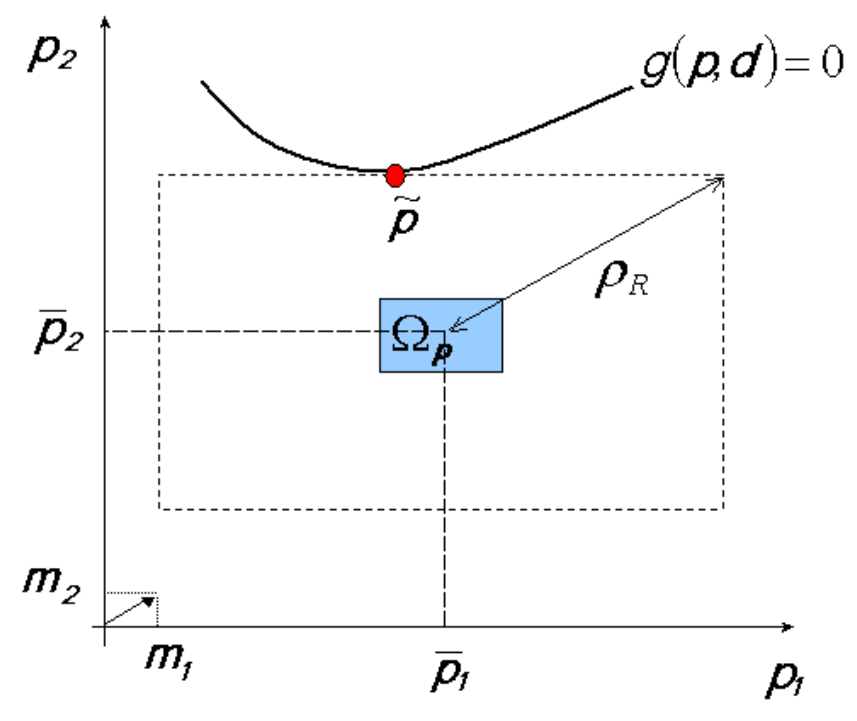

Figure 1. Relevant metrics for a rectangular Reference Set.

$\mathcal{M}_{p}^{c}$ of $\mathcal{M}_{p}$ bounds the Failure Domain. On the other hand, when the constraints are not satisfied at the Designated Point, the complement of $\mathcal{M}_{\boldsymbol{p}}$ bounds the non-failure domain.

The notions and metrics introduced above do not require probabilistic uncertainty models. Since uncertainty models can be probabilistic in nature, one may wonder how to integrate such models with the above developments. Recall that when constraints are feasible, the failure probability is equal to zero. When the complement set of $\mathcal{M}_{\boldsymbol{p}}$ bounds the Failure Domain, an upper bound to the failure probability can be easily calculated. This bound is given by

$$
\psi_{\boldsymbol{p}}=1-P\left[\mathcal{M}_{\boldsymbol{p}}\right]=P\left[\mathcal{M}_{\boldsymbol{p}}^{c}\right]
$$

where $P[\cdot]$ is the probability operator. If the components of $\boldsymbol{p}$ are independent random variables and $\Omega_{\boldsymbol{p}}$ is the hyper-rectangle $\mathcal{R}(\overline{\boldsymbol{p}}, \boldsymbol{m})$, Equation (9) leads to

$$
\psi_{\boldsymbol{p}}=1-\prod_{i=1}^{\operatorname{dim}(\boldsymbol{p})}\left[F_{\boldsymbol{p}_{i}}\left(\overline{\boldsymbol{p}}_{i}+\frac{\rho_{R} \boldsymbol{m}_{i}}{\|\boldsymbol{m}\|}\right)-F_{\boldsymbol{p}_{i}}\left(\overline{\boldsymbol{p}}_{i}-\frac{\rho_{R} \boldsymbol{m}_{i}}{\|\boldsymbol{m}\|}\right)\right],
$$

where $F_{\boldsymbol{p}_{i}}(\boldsymbol{p})$ is the Cumulative Distribution Function (CDF) of $\boldsymbol{p}_{i}$. Note that there is an upper bound for each non-proportional Reference Set. While the bound corresponding to $\Omega_{\boldsymbol{p}}=\mathcal{R}\left(\overline{\boldsymbol{p}}^{(1)}, \alpha \boldsymbol{m}^{(1)}\right)$ is independent of $\alpha$, the bounds that result from finding the Maximal 
Feasible Homothets of $\mathcal{R}\left(\overline{\boldsymbol{p}}^{(1)}, \boldsymbol{m}^{(1)}\right), \mathcal{R}\left(\overline{\boldsymbol{p}}^{(2)}, \boldsymbol{m}^{(1)}\right)$, and $\mathcal{R}\left(\overline{\boldsymbol{p}}^{(1)}, \boldsymbol{m}^{(2)}\right)$ can all be different. The main advantage of using Equation (9) is that failure probability bounds corresponding to new probabilistic uncertainty models are trivial to evaluate since they do not require solving for $\mathcal{M}_{\boldsymbol{p}}$ again. As compared to methods based on the transformation of the problem to standard normal space, this bound not only avoids the numerical error generated by the transformation (which ranges from considerable to unmanageable as failure probabilities become smaller) but also greatly reduces most of the dependence of the calculations on the uncertainty model assumed. It should be noted that, using Equation (9) also has a downside. Since the $\boldsymbol{p}$-deformation needed to size $\mathcal{M}_{\boldsymbol{p}}$ does not use the probabilistic uncertainty model, the upper bound $\psi_{\boldsymbol{p}}$ might be large. Better bounds in Equation (9) are obtained if $P\left[\mathcal{M}_{\boldsymbol{p}}\right]$ can be increased by variation of the geometry of the Reference Set. Strategies for this are considered in Section IV.B.

\section{Constraint Feasibility}

It is necessary to find an algorithmic tool to implement the constraint feasibility condition

$$
\boldsymbol{g}(\boldsymbol{p}, \boldsymbol{d}) \leq \mathbf{0} \text { for all } \boldsymbol{p} \in \Delta_{\boldsymbol{p}}
$$

in Problem 2. Determining constraint feasibility is not a simple task since there are an infinite number of parameter realizations for which the constraints must be satisfied. Since the numerical search for a solution to the robust optimization problem requires repeated determination of constraint feasibility, the test must also be efficient. For use in standard constrained optimization software, this test should be in the form of an inequality constraint. Tools commonly used for uncertainty analysis and design, such as Monte Carlo Sampling (MCS) and the First Order Reliability Method (FORM), ${ }^{13}$ are not well-suited to determine constraint feasibility. More importantly, they are ineffective and inconclusive when dealing with cases where constraints are feasible. In this case, with all points in the Uncertainty Set being feasible, the optimization used in FORM diverges since, in standard normal space, the limit state function has no level curves at the zero level; while the error due to finite sample size in the MCS estimate of the probability of constraint violation might wrongly indicate constraint feasibility. Since Problem 2 precisely targets design points at which there are no constraint violations, and therefore these numerical tools break down, the need for constraint feasibility tests is apparent.

11 of 25

American Institute of Aeronautics and Astronautics 
The means by which the constraints in Problem 2 are to be implemented will be provided by a constraint feasibility test. Constraint feasibility tests have been recently proposed ${ }^{7}$ by the authors. Such tests not only allow determination of constraint feasibility condition for hyper-spherical and hyper-rectangular uncertainty sets, but also make Problem 2 computationally tractable.

The constraint feasibility test is based on the comparison of $\mathcal{M}_{\boldsymbol{p}}(\boldsymbol{d})$ with the uncertainty set $\Delta_{p}$. If the Feasibility Condition $\Delta_{p} \subseteq \mathcal{M}_{\boldsymbol{p}}(\boldsymbol{d})$ holds, the constraints are feasible, i.e., for this fixed $\boldsymbol{d}, \boldsymbol{g}(\boldsymbol{p}, \boldsymbol{d}) \leq \mathbf{0}$ for all $\boldsymbol{p} \in \Delta_{\boldsymbol{p}}$. On the other hand, constraints are infeasible if there exist a parameter realization for which at least one constraint is violated. For arbitrarily ${ }^{6}$ shaped uncertainty sets, many feasible design points may be captured by using a hyperspherical or a hyper-rectangular set to bound $\Delta_{p}$, and comparing the size of the bounding set with that of the Maximal Feasible Homothet. The constraint condition in Problem 2 is implemented either exactly (if $\Delta_{p}$ is a hyper-sphere or hyper-rectangle) or conservatively (if $\Delta_{p}$ must be bounded by a hyper-sphere or hyper-rectangle). The feasibility condition is realized as an inequality constraint by comparing the sizes of the uncertainty set (or its bound) with the Maximal Feasible Homothet; e.g. if $R$ is the radius of the bounding set, $\rho_{R}(\boldsymbol{d})$ is the Spherical PSM of the Maximal Feasible Homothet, and $\rho_{R}(\boldsymbol{d}) \geq R$, the constraints are feasible for this $\boldsymbol{d}$.

The design space can be explored by determining if $\Delta_{p} \subseteq \mathcal{M}_{p}(\boldsymbol{d})$ as $\boldsymbol{d}$ varies. If a design point for which $\Delta_{p} \subseteq \mathcal{M}_{\boldsymbol{p}}(\boldsymbol{d})$ is found, a solution to Problem 2 exists. The set of designs for which this is attained is exactly the RDS if the uncertainty set is hyper-spherical or hyper-rectangular, and is a conservative approximation to the RDS if the uncertainty set must be bounded by a hyper-sphere or hyper-rectangle. The design point for which the PSM is maximal is called the Robustness Driven Design. For this design, the corresponding Maximal Feasible Homothet is the largest. If constraints are infeasible for all design points, say because the CPV corresponding to the Robustness Driven Design is an interior point of $\Delta_{p}$, a solution to Problem 2 does not exist.

\section{IV.A. Optimality Criteria}

In this section, it is assumed that constraints are feasible for at least one design point. Therefore, a solution to Problem 2 exists. A few candidates for the design criterion functional $J$ for use in Problem 2 are introduced next. Recall that once the uncertainty set $\Delta_{p}$ and the performance function $y$ are fixed, the uncertainty set $\Delta_{y}(\boldsymbol{d})=y\left(\Delta_{\boldsymbol{p}}, \boldsymbol{d}\right)$ is determined by the 
design parameter $\boldsymbol{d}$, and so the value of $J$ acting on that uncertainty set is determined by $\boldsymbol{d}$. Thus, solving Problem 2 can be considered to be optimizing $J(\boldsymbol{d})$ over the set of feasible designs $\boldsymbol{d}$.

\section{IV.A.1. Worst-Case}

This criterion is to be used when we want to minimize the worst-case performance value. The corresponding cost functional is given by

$$
J_{W C} \triangleq y\left(\boldsymbol{p}^{*}, \boldsymbol{d}\right)
$$

where

$$
\boldsymbol{p}^{*}=\underset{\boldsymbol{p}}{\operatorname{argmax}}\left\{y: \boldsymbol{p} \in \Delta_{\boldsymbol{p}}\right\}
$$

is the Worst-Case Parameter Realization. In the above equation is assumed that the smaller the value of $y$ the better the corresponding design. If the opposite holds, use the $\operatorname{argmin}\{\cdot\}$ operator in Equation (12). In general, the Worst-Case Parameter Realization depends on the design variable and might not correspond to the parameter realizations that are the furthest from the Designated Point. Therefore, the search for an optimal design in the worst-case sense requires solving a min-max problem. Obviously, the implementation of this criterion requires that $\Delta_{y}$ be bounded from above for all the design points within the RDS. This requirement imposes restrictions on the structure of $y(\boldsymbol{p}, \boldsymbol{d})$ within the support set $\Delta_{\boldsymbol{p}}$. Optimal designs corresponding to this criterion provide performance guarantees, since all other realizations of $y$ are less or equal than $J_{W C}$. However, its use may lead to excessively conservative designs since the Worst-Case Parameter Realization might be extreme and very unlikely. Strategies to identify ${ }^{1}$ and approximate ${ }^{16,17}$ this realization are available. Since the implementation of this criterion only requires prescribing the support set of the uncertain parameter, probabilistic uncertainty models are redundant.

\section{IV.A.2. Least-Second-Moment}

The implementation of this criterion requires a probabilistic uncertainty model. This criterion is to be used when we want to concentrate all possible performance values as close as possible to a target value. We might want this to improve performance reproducibility or to 
reduce its variability. The corresponding cost functional is given by

$$
J_{L S M} \triangleq \mathrm{V}[y]+\mathrm{E}[y](\mathrm{E}[y]-2 \hat{y})+\hat{y}^{2},
$$

where $\mathrm{E}[\cdot]$ and $\mathrm{V}[\cdot]$ are the expected value and the variance operators respectively and $\hat{y}$ is a target value. Equation (13) results from calculating the second moment of the random performance metric about the target value. By minimizing $J_{L S M}$ in Problem 2, we are searching for a design point that maximizes the concentration of $y$ about $\hat{y}$, i.e. in an ideal case, the optimal design leads to $f_{y}(y)=\delta(y-\hat{y})$, where $\delta(\cdot)$ is the Dirac delta. The minimization of the variability of $y$ can be obtained from Equation (13) by substituting $\mathrm{E}[y]$ for $\hat{y}$. This is not quite a special case, since in general $\mathrm{E}[y]$ is a function of $\boldsymbol{d}$, and $\hat{y}$ is not.

\section{IV.A.3. Reliability-based}

As before, the implementation of this criterion requires a probabilistic uncertainty model. This criterion is to be used when we want the performance to stay within a prescribed admissible range. The corresponding cost functional is given by

$$
J_{R E_{1}} \triangleq P[(y<\underline{y}) \cup(y>\bar{y})]+h(\underline{y}, \bar{y}),
$$

where $y$ and $\bar{y}$ are the minimum and maximum allowable performance values respectively, and $h$ is a penalty function. The variables $\underline{y}, \bar{y}$, and the function $h$ are prescribed in advance. Equation (14) allows for using shapable Failure Domains by augmenting the set of design variables. See the authors' prior work ${ }^{11}$ for details. Thanks to the first term in Equation (14), the probability of attaining performance values within the non-failure domain $\underline{y} \leq$ $y \leq \bar{y}$ is maximized. The last term can be used to enhance the resulting performance by simultaneously reducing $\bar{y}-\underline{y}$. For instance, if $y$ is the weight of the structure, assuming $\underline{y}=0$, and $\bar{y}=e$, implies that the admissible weight range is $[0, e]$. By using Equation (14) with $h(e)=e / y_{\max }$ for $e>0$, and making $e$ an additional design variable in Problem 2 , the size of the admissible weight range is reduced while the probability of $y$ exceeding $e$ is simultaneously minimized. The parameter $y_{\max }$ is chosen by the designer to balance the influence of the two terms in Equation (14).

Note that violations to the requirements $\boldsymbol{g} \leq \mathbf{0}$ were regarded as unacceptable, i.e., these constraints are "hard". Violations to $\underline{y} \leq y \leq \bar{y}$ in Equation (14) are allowed; so these constraints, which are built into the design criterion functional $J$, are "soft". This 
distinction must be taken into account when deciding in which of these two formats a given requirement should be cast.

The numerical tools available for the estimation of failure probabilities might be inaccurate (e.g. FORM for a large failure probability), computationally expensive (e.g. sampling for a small failure probability) or unsuitable for gradient-based design optimization (e.g. the estimation of $J_{R E}$ via sampling leads to a piecewise constant function of $\boldsymbol{d}$ ). The upper bounds in Equation (9) provide an alternative way to enforce the reliability-based design criterion. There are several advantages to using upper bounds in an optimization driven design procedure, instead of using the available estimates of failure probability.

(a) The calculation of the upper bound is relatively cheap compared to estimating failure probability by Monte Carlo sampling.

(b) The upper bound is a true conservative overestimate of the failure probability, while there is no way of knowing whether estimates of failure probability by FORM or Monte Carlo underestimate or overestimate the value.

(c) The accuracy of the upper bound does not depend on the value of the failure probability, while the accuracy of both FORM and Monte Carlo sampling with a fixed number of sample points is very sensitive to the actual value of the failure probability.

(d) These bounds are smooth functions of the design variable, whereas a Monte Carlo estimate of failure probability using a fixed sample is a piecewise constant function of the design variable with step discontinuities as changing the design variable makes the failure boundary move across any of the sample points.

Using the bounds, Equation (14) is replaced by

$$
J_{R E_{2}} \triangleq \psi_{\boldsymbol{p}}+h(\underline{y}, \bar{y})
$$

where $\psi_{\boldsymbol{p}}$ results from using Equation (9) along with the constraint function $\boldsymbol{g}=[y-\bar{y}, \underline{y}-$ $y] \leq \mathbf{0}$. Note that $J_{R E_{1}} \leq J_{R E_{2}}$.

\section{IV.B. Infeasible Constraints}

This section considers the case when one or several of the constraints in $\boldsymbol{g}$ are infeasible. If the design requirements can not be relaxed (i.e. the way in which $\boldsymbol{g}$ depends on $\boldsymbol{p}$ is 
fixed), and the uncertainty model cannot be refined, the corresponding requirements must be enforced as soft constraints. This practice implies that (some, presumably limited) constraint violations are now acceptable. One may decide to only use this format after realizing that since no uncertainty model is perfect, the value of attaining constraint feasibility is mostly mathematical. Strategies for the efficient implementation of the soft constraints are presented next.

The ideas that follow require probabilistic uncertainty models for their implementation. Since the requirements corresponding to the constraints in Problem 2 have been regarded as important, the performance metric $y$ will now be ignored. The emphasis is now on finding a design which is as close to feasible as possible. If the constraint vector is written as $\boldsymbol{g}=\left[\boldsymbol{g}_{i}, \boldsymbol{g}_{f}\right]$, where $\boldsymbol{g}_{i}$ has the infeasible constraints and $\boldsymbol{g}_{f}$ has the feasible ones, a reliabilitybased formulation to the problem of interest is

$$
\min _{\boldsymbol{d}}\left\{P\left[\boldsymbol{g}_{i} \not \leq \mathbf{0}\right]: \boldsymbol{g}_{f} \leq \mathbf{0}, \forall \boldsymbol{p} \in \Delta_{\boldsymbol{p}}\right\}
$$

The inequality $\boldsymbol{g}_{i} \not \leq \mathbf{0}$ says that some component of the vector $\boldsymbol{g}_{i}$ is positive which is on the failure side of the constraint boundary, so the event represented by $\left[\boldsymbol{g}_{i} \not \leq \mathbf{0}\right]$ is the $\boldsymbol{g}_{i}$ failure set.

It was noted earlier that the upper bound on failure probability could be improved by changing the geometry of the Reference Set. Another means of looking for improved upper bounds is by transforming the uncertain parameters to become random variables with different distributions. A commonly used and well studied transformation results in the transformed parameters being an independent vector of standard normal random variables, denoted by $\boldsymbol{u}$. The transformation ${ }^{13}$ between $\boldsymbol{p}$-space and $\boldsymbol{u}$-space will be denoted as $\boldsymbol{u}=$ $U(\boldsymbol{p})$.

The developments in prior work ${ }^{14,15}$ are based on the homothetic deformation of hyperspheres and hyper-rectangles in $\boldsymbol{u}$-space. In that work, ideas parallel to the ones introduced in Section III are developed. For instance, analogous to the Spherical PSM is the Spherical Reliability Index $\beta_{S}$, and to the Rectangular PSM is the Rectangular Reliability Index $\beta_{R}$. Likewise the CPV and the Maximal Feasible Homothet in $\boldsymbol{p}$-space are analogous to their counterparts in $\boldsymbol{u}$-space. The Reliability indices (RI) are the best measure of robustness of a given design for a given uncertainty model providing that such a design is non-robust.

16 of 25

American Institute of Aeronautics and Astronautics 
Analogous to Equation (9) is the upper bound to the failure probability

$$
\psi_{\boldsymbol{u}}=1-P\left[\mathcal{M}_{\boldsymbol{u}}\right]=P\left[\mathcal{M}_{\boldsymbol{u}}^{c}\right],
$$

where $\mathcal{M}_{\boldsymbol{u}}$ is the Maximal Feasible Homothet resulting from the deformation in $\boldsymbol{u}$-space. Note that bounds in Equations (14) and (16) can be calculated using Equation (17) as well. In contrast to $\psi_{\boldsymbol{p}}, \psi_{\boldsymbol{u}}$ can be calculated analytically for both hyper-spherical and hyperrectangular Reference Sets. Expressions for this can be found in the an earlier paper. ${ }^{15}$ The unimodal, rotationally symmetric, and exponentially decreasing nature of the PDF in $\boldsymbol{u}$ space allows for placing hyper-spherical and hyper-rectangular Reference Sets so that their Maximal Feasible Homothets capture more success domain probability. This practice may yield better upper bounds than the ones in Equation (9). However, the calculation of $\psi_{\boldsymbol{u}}$ is strongly dependent on the uncertainty model assumed.

Deformations in $\boldsymbol{u}$-space also enable accurate and efficient approximations to the failure probability. ${ }^{15}$ Note that the bounds $\psi_{\boldsymbol{p}}$ and $\psi_{\boldsymbol{u}}$ are probabilities of events, so they fall in the numerical range of zero to one. The calculation of $\psi_{\boldsymbol{p}}$ and $\psi_{\boldsymbol{u}}$ enables the approximation of the set of designs for which the failure probability is below an admissible value. This information greatly facilitates the implementation of design optimization schemes.

\section{Example}

A two-dimensional problem in the design variables, i.e., $\boldsymbol{d}=\left[\boldsymbol{d}_{1}, \boldsymbol{d}_{2}\right]$, and in the uncertain parameter, i.e., $\boldsymbol{p}=\left[\boldsymbol{p}_{1}, \boldsymbol{p}_{2}\right]$, has been selected to facilitate visualization of the concepts introduced in this paper. The same example has been used in previous work ${ }^{6,7,14,15}$ to exemplify other aspects of the methodology. It is assumed that the Designated Point is $\overline{\boldsymbol{p}}=[1,1]$, while the performance metric and the constraint set are

$$
\begin{aligned}
& y=\left(\boldsymbol{d}_{1}+\frac{39 \boldsymbol{p}_{1}}{5}-9 \boldsymbol{p}_{2}\right)^{2}+\left(\boldsymbol{d}_{2}+7 \boldsymbol{p}_{1}-\frac{87 \boldsymbol{p}_{2}}{10}\right)^{2} \\
& \boldsymbol{g}=\left[\begin{array}{c}
3 \boldsymbol{d}_{2}-4 \boldsymbol{p}_{1}^{2}-4 \boldsymbol{d}_{1} \boldsymbol{p}_{2} \sin \left(\boldsymbol{p}_{2} \boldsymbol{d}_{1}-\boldsymbol{p}_{1}^{2}\right) \\
-\sin \left(\boldsymbol{p}_{1}^{2} \boldsymbol{p}_{2}-\sin \left(2 \boldsymbol{p}_{1}-2\right)\right)-\boldsymbol{d}_{1} \boldsymbol{d}_{2} \boldsymbol{p}_{1}-\boldsymbol{p}_{2} \\
\boldsymbol{d}_{1}+\boldsymbol{p}_{1}^{2} \boldsymbol{d}_{2}^{2}-4 \boldsymbol{p}_{2}^{2} \boldsymbol{p}_{1}-4 \sin \left(2 \boldsymbol{p}_{1}-2 \boldsymbol{p}_{2}\right) \\
2\left(\boldsymbol{p}_{1}+\boldsymbol{p}_{2}\right) \sin \left(\boldsymbol{p}_{1}^{2}-\boldsymbol{d}_{2}\right)-2 \boldsymbol{p}_{1} \boldsymbol{p}_{2}\left(\boldsymbol{d}_{2}+2 \boldsymbol{p}_{1}^{2}-2\right)+\boldsymbol{d}_{1}-6 \boldsymbol{p}_{1}
\end{array}\right]
\end{aligned}
$$

17 of 25 
The PSMs for design points within the FDS corresponding to $\Omega_{\boldsymbol{p}}=\mathcal{S}(\overline{\boldsymbol{p}}, R)$ are shown in Figure 2. In this example, the spherical PSM can be interpreted as the largest percentile of variation in $\boldsymbol{p}$ from its nominal value allowed before a constraint is violated. According to the figure, parameter variations of less than $53 \%$ lead to non-empty RDSs. Therefore, the Robustness Driven Design is able to tolerate variations of up to $53 \%$ in $\boldsymbol{p}$. Notice that more than one PSM maximum occurs (there are local maxima in the vicinity of $[-1,0]$ and of $[-2.2,-2.4])$. More importantly, notice that designs further in from the boundary of the FDS are not necessarily more robust. For instance, the comparison of $\boldsymbol{d}^{(1)}=[-2.25,-2.4]$ and $\boldsymbol{d}^{(2)}=[-1,-1]$ shows that $\rho_{S}\left(\boldsymbol{d}^{(1)}\right) \gg \rho_{S}\left(\boldsymbol{d}^{(2)}\right)$ even though $\boldsymbol{d}^{(1)}$ is much closer to a constraint limiting the FDS than $\boldsymbol{d}^{(2)}$. Figure 3 shows the rectangular PSM values for $\Omega_{\boldsymbol{p}}=\mathcal{R}(\overline{\boldsymbol{p}},[1,4])$.

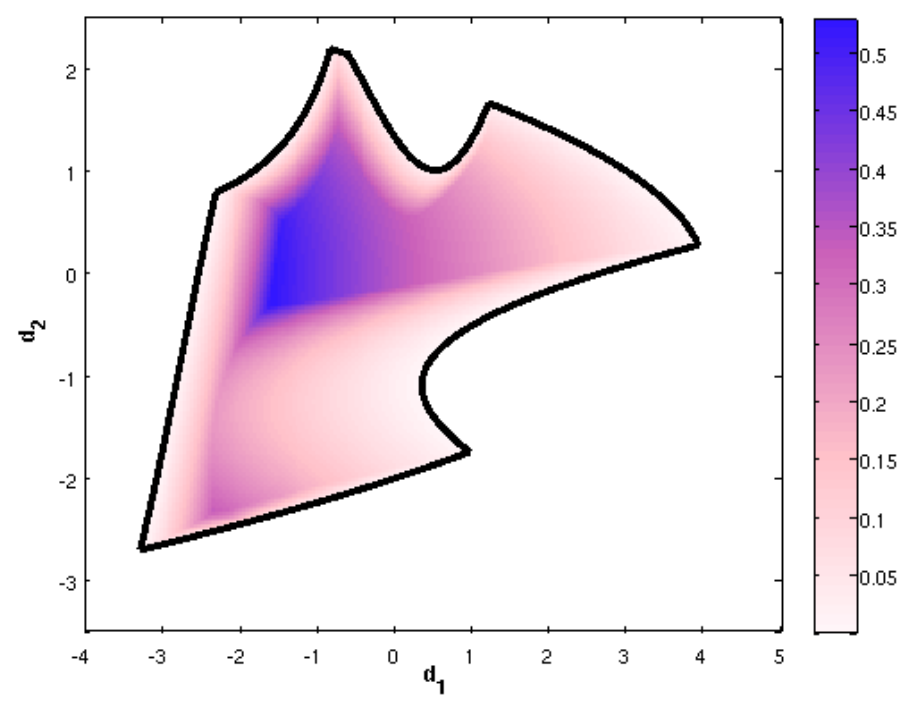

Figure 2. Spherical PSM $\rho_{S}(d)$ in the FDS.

Considerable differences in the distribution and magnitude of the PSMs, as compared to the previous figure, are apparent. This indicates a strong dependence of the robustness on the geometry of the Reference Set. Consider the Support Set $\Delta_{p}(\mu)=\mathcal{R}(\overline{\boldsymbol{p}}, \mu[1,4])$. When $\mu$ assumes the value of zero, the Problems 1 and 2 are identical since there is no uncertainty, and all the $J_{\mathrm{s}}$ of Section IV.A coincide. The solution to this problem will be referred to as the Deterministic Optimal Design. From Figure 4 it can be seen that two constraints are active at the Deterministic Optimal Design point. Increasing values of $\mu$ in $\Delta_{p}(\mu)$ will now be assumed. For each value of $\mu$, it is assumed that $\boldsymbol{p}_{1}$ and $\boldsymbol{p}_{2}$ are independent random 


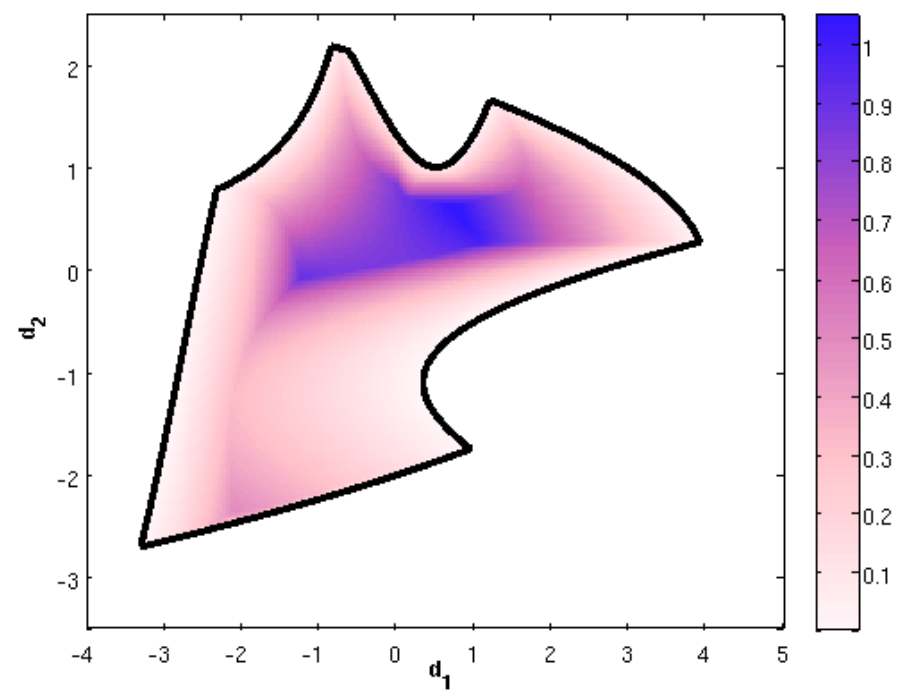

Figure 3. Rectangular PSM $\rho_{R}(\boldsymbol{d})$ in the FDS.

variables which have Beta distributions based on $\beta(5,2)$ and $\beta(2,5)$, respectively, but shifted and scaled to be supported by $\Delta_{p}(\mu)$.

For each $\mu$ value, solutions that minimize the cost functionals in Equations (11-14) are calculated. Since the performance function $y$ is non-negative, the best possible performance value that any design could attain is zero. By using the worst-case criterion one intends to identify the design which minimizes the largest $y$. By using the least-second-moment criterion, one tries to concentrate $y$ near the target $\hat{y}=0$. By assuming $\bar{y}=(\max \{y\}+$ $3 \min \{y\}) / 4, \underline{y}=-\infty$ and $h=\bar{y}$, when using this criterion, one tries to reduce the probability that $y$ lies in the upper three-quarters of $\Delta_{y}$ while reducing the value of the number which marks the division of the lower quarter and the upper three-quarters of the $\Delta_{y}$. Note that $\Delta_{y}, \bar{y}$ and $h$, are functions of the design parameter. The worst-case optimal designs for increasing values of $\mu$ are grouped in a set referred to as the optimal trail in the worst-case sense. Optimal trails corresponding to the least-second-moment and the reliability-based design criteria are also generated. These three trails are shown in Figure 4. Moving along a design trail from the Deterministic Optimal Design to the designs corresponding to increasing values of $\mu$ corresponds to optimizing the corresponding cost functional over increasingly larger uncertainty sets. Note that the three trails converge to the Robustness Driven Design at $\mu^{*}=0.26$, which is $\boldsymbol{d}^{*}=[0.718,0.631]$. Recall that the Robustness Driven Design is the 
one for which the PSM is the largest. In this case the uncertainty set has grown so large that the RDS is reduced to a point, therefore all three cost functionals lead to $\boldsymbol{d}^{*}$.

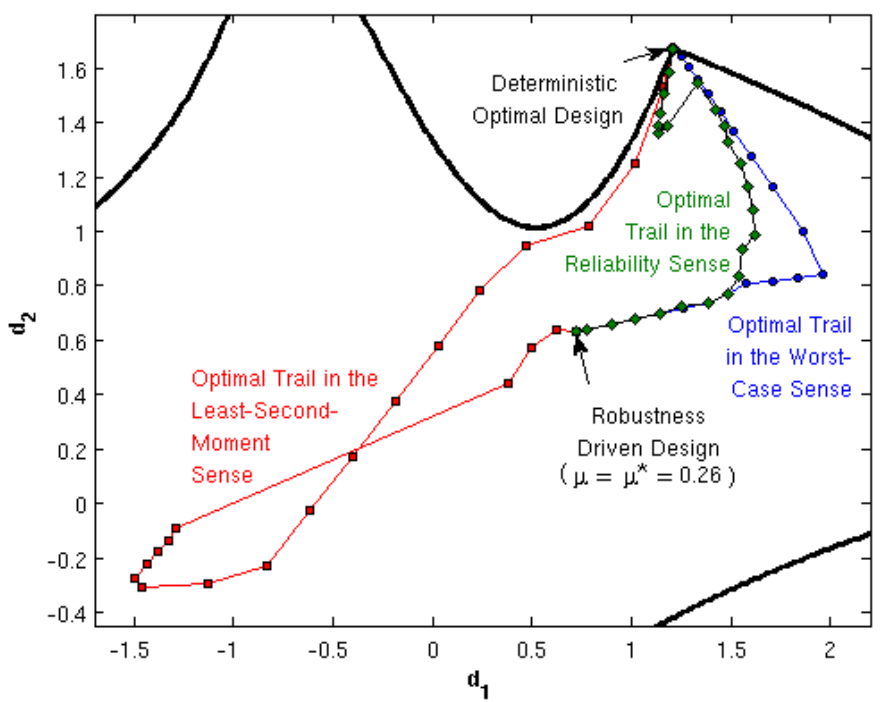

Figure 4. Optimal trails.
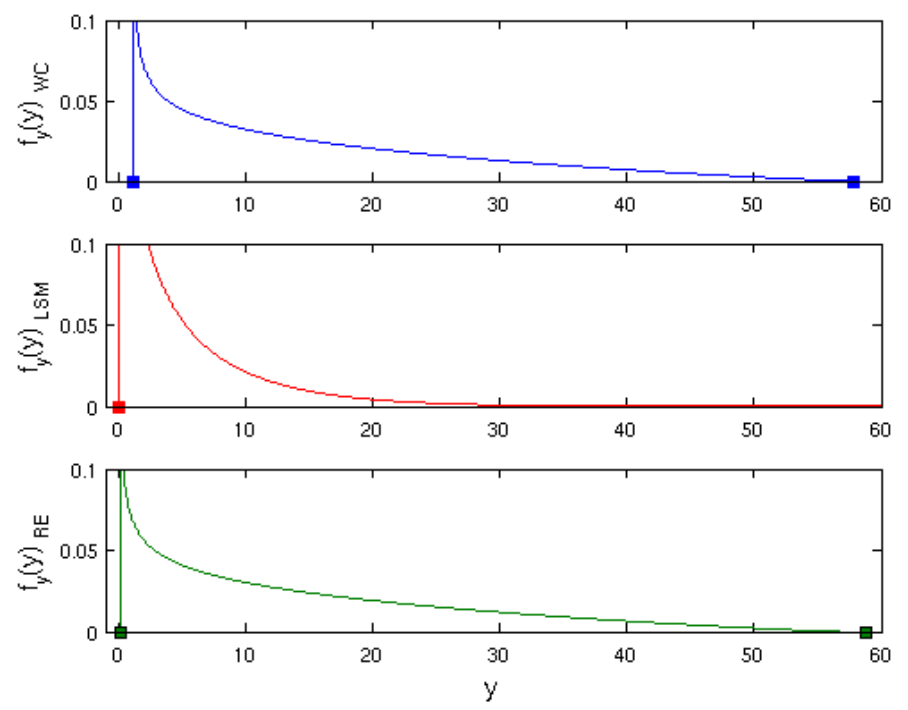

Figure 5. Optimal PDFs for $\mu=0.1235$.

The PDFs of the optimal performance functions corresponding to $\mu=0.1235$ are shown 
in Figure 5. The corresponding support sets are $\Delta_{y}=[1.242,57.85]$ for the worst-case criterion, $\Delta_{y}=[0,105.63]$ for the least-second-moment criterion and $\Delta_{y}=[0.197,58.81]$ for the reliability-based criterion. Notice that the PDF for the worst-case solution does not cover the vicinity of the ideal value of $y=0$. This is not the case for the other two solutions.

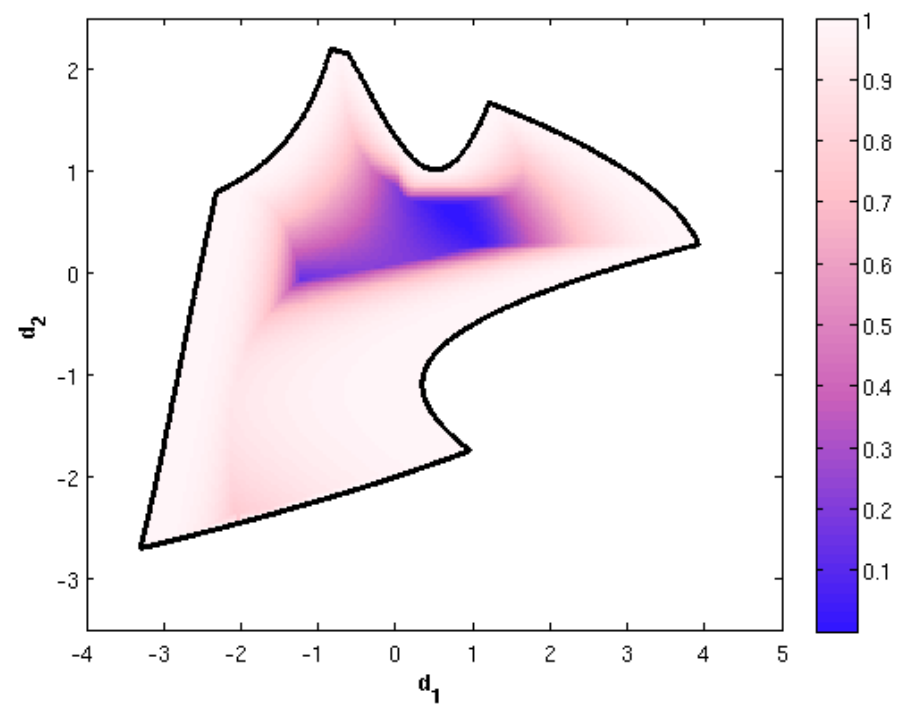

Figure 6. $\psi \boldsymbol{p}(\boldsymbol{d})$ in the FDS for $\Omega \boldsymbol{p}=\mathcal{R}(\overline{\boldsymbol{p}},[1,4])$.

In what follows we assume $\mu=0.26004$ in the probabilistic uncertainty model of $\boldsymbol{p}$ defined earlier. Since $\mu>\mu^{*}$, at least one constraint is infeasible, the RDS does not exist, and $P[\mathcal{F}]>0$ for all design points. The functions $\psi_{\boldsymbol{p}}(\boldsymbol{d})$ and $\psi_{\boldsymbol{u}}(\boldsymbol{d})$ corresponding to the Reference Sets $\Omega_{\boldsymbol{p}}=\mathcal{R}(\overline{\boldsymbol{p}},[1,4])$, and $\Omega_{\boldsymbol{u}}=\mathcal{R}(\mathbf{0},[1,1])$ are considered next. The bound $\psi_{\boldsymbol{p}}$ is shown in Figures 6 and 7. While Figure 6 clearly shows large bound values, Figure 7 is used to better illustrate small ones. The resolution used in this figure does not allow to see the smallest bound, which is $\psi_{\boldsymbol{p}}\left(\boldsymbol{d}^{*}\right)=2.4972 \times 10^{-10}$. Recall that the calculation of $\psi_{\boldsymbol{p}}$ for a different uncertainty model would only require changing the CDF in Equation (10).

Figures 8 and 9 show $\psi_{\boldsymbol{u}}(\boldsymbol{d})$. The contour of the FDS corresponding to $\psi_{\boldsymbol{p}}(\boldsymbol{d})$ is shown as a dashed line in Figure 8. The two FDSs are different since the Reference Sets use different Designated Points, i.e., $\overline{\boldsymbol{p}} \neq U^{-1}(\mathbf{0})$. The smallest $\psi_{\boldsymbol{u}}(\boldsymbol{d})$ bound, which corresponds to the design point attaining the largest Rectangular RI, which is $\beta_{\mathcal{R}}=12.22$, is $\psi_{\boldsymbol{u}}\left(\boldsymbol{d}^{\dagger}\right)=$ $4 \Phi(-8.6408) \Phi(8.6408)=1.1160 \times 10^{-17}$, where $\Phi$ is the Cumulative Distribution Function 


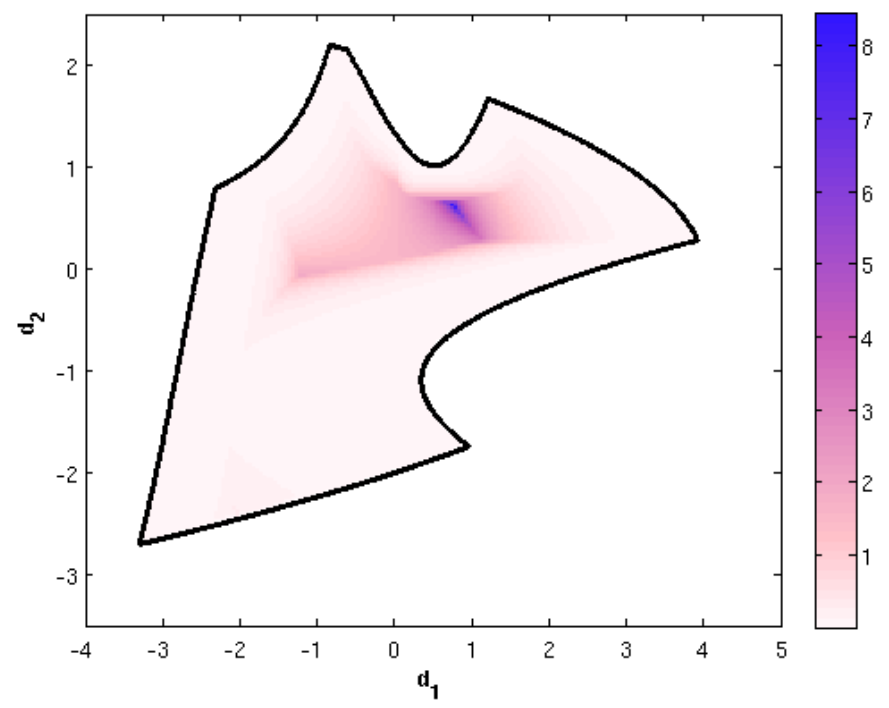

Figure 7. $-\log \left(\psi_{\boldsymbol{p}}(\boldsymbol{d})\right)$ in the FDS for $\Omega_{\boldsymbol{p}}=\mathcal{R}(\overline{\boldsymbol{p}},[1,4])$.

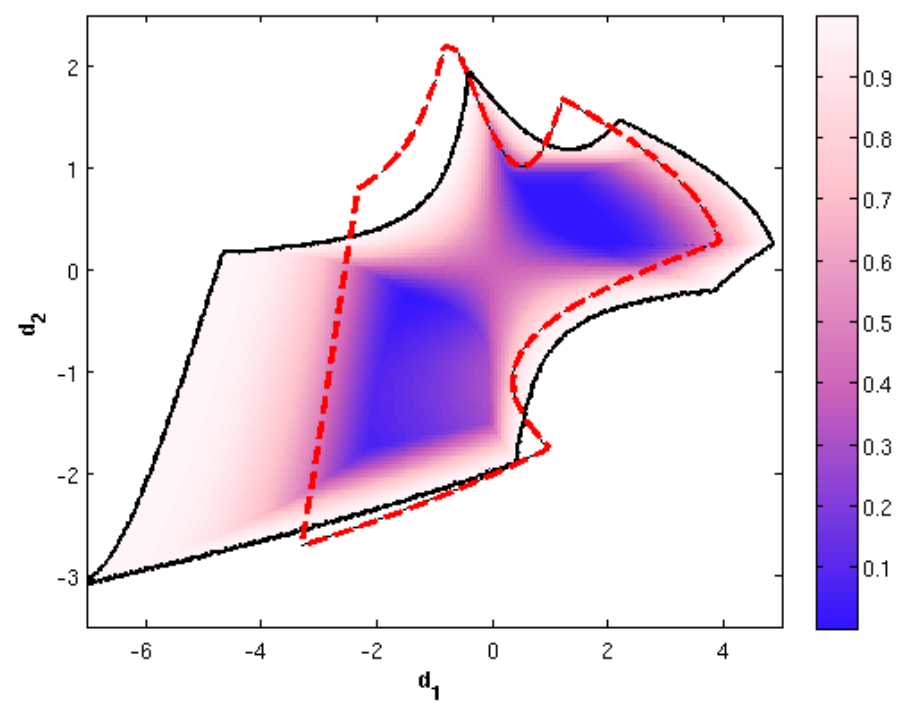

Figure 8. $\psi \boldsymbol{u}(\boldsymbol{d})$ in the FDS for $\Omega \boldsymbol{u}=\mathcal{R}(0,[1,1])$

of a standard normal random variable and $\boldsymbol{d}^{\dagger}$ is approximately equal to $\boldsymbol{d}^{*}$. As before, the resolution of the figures prevents us from seeing this value. This bound is so small that the evaluation of the equivalent expression $1-(\Phi(8.6408)-\Phi(-8.6408))^{2}$ is approximated with 


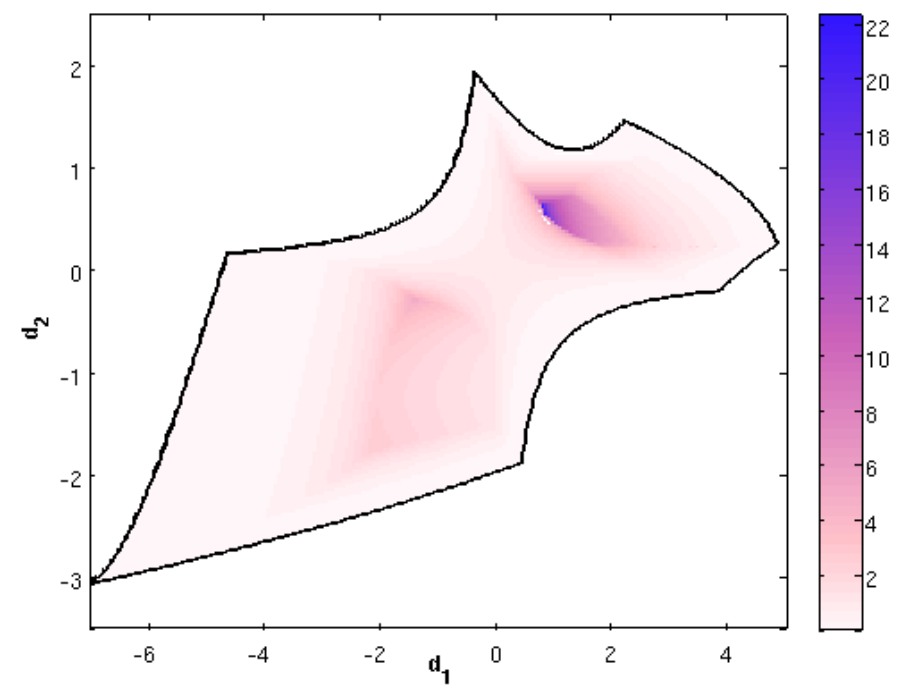

Figure 9. $-\log (\psi \boldsymbol{u}(\boldsymbol{d}))$ in the FDS for $\Omega \boldsymbol{u}=\mathcal{R}(\mathbf{0},[1,1])$.

0 by Matlab ${ }^{\circledR}$. In general, the designs $\boldsymbol{d}^{*}$ and $\boldsymbol{d}^{\dagger}$ are different. In this example, $\boldsymbol{d}^{\dagger} \approx \boldsymbol{d}^{*}$ since $\Delta_{p}$ and $\mathcal{M}_{p}\left(\boldsymbol{d}^{*}\right)=\mathcal{R}\left(\overline{\boldsymbol{p}}, \mu^{*}[1,4]\right)$ are almost identical. Since the corresponding Reference Set is anchored at the origin of $\boldsymbol{u}$-space, where most of the probability is, considerably smaller bounds are obtained as compared to the ones in Figures 6-7. Recall that for a different uncertainty model, and in contrast to $\psi_{\boldsymbol{p}}$, the calculation of $\psi_{\boldsymbol{u}}$ requires solving a new optimization problem to identify the new the Maximal Feasible Homothet.

There were instances during the calculation of $\psi_{\boldsymbol{u}}(\boldsymbol{d})$ when the numerical error caused by the transformation to $\boldsymbol{u}$-space $U(\boldsymbol{p})$, led to inaccurate results. This occurs when $\|\boldsymbol{u}\| \gg 0$. Due to this numerical anomaly, the $\boldsymbol{u}$-space must be artificially bounded when searching for the CPV. Failing to impose these bounds may result in the numerical search not converging. The usage of such bounds, however, imposes a low numerical limit on the values of $\psi_{\boldsymbol{u}}$ that can be calculated. Notice that lower values of $\psi_{\boldsymbol{p}}(\boldsymbol{d})$ would have been obtained if we chose $\overline{\boldsymbol{p}}=U^{-1}(\mathbf{0})$ instead. Such practice, however, imposes the need for a probabilistic uncertainty model. In the absence of a such a model, a natural choice for the Designated Point is the geometric center of the uncertainty set. 


\section{Concluding Remarks}

Strategies for the analysis and solution to robust optimization problems are proposed herein. The methodology developed provides the means to quantify the robustness characteristics of a given design point according to existing levels of uncertainty. In addition, it allows for determining constraint feasibility in a conclusive and computationally tractable manner. Strategies to deal with feasible and infeasible constraints are considered. This paper implements worst-case, least-second-moment and reliability-based design criteria into the robust optimization problem of interest. When the worst-case design criterion is applicable, performance guarantees are attainable. However, such solutions are usually driven by extreme and very unlikely parameter realizations, resulting in designs with excessive conservatism. In contrast, design criteria using probabilistic uncertainty models are able to account for the likelihood of all possible outcomes. In this setting, a reliability-based design captures the intent of the worst-case criterion since extreme parameter realizations still drive the design process. However, the amount of conservatism is considerably reduced since it allows for some realizations to fall outside the admissible range of performance. In contrast to these two criteria, a least-second-moment design is driven by the most likely events. This characteristic is appealing from the practical point of view, esspecially when the resulting probability of unfavorable performance is low. Upper bounds to failure probability, based on the homothetic deformation of sets in the original uncertain space, were proposed herein. Design-optimization schemes based on these bounds have several advantages: the calculation of the bounds is comparatively cheap, their usage considerably relaxes the dependence of the calculations on the uncertainty model assumed, and their values are guaranteed to bound the failure probability from above.

\section{References}

${ }^{1}$ Darligton, J., Pantelides, C., Rustem, B., and Tanyi, B., "An algorithm for constrained nonlinear optimization under uncertainty," Automatica, Vol. 35, 1999, pp. 217-228.

${ }^{2}$ Mulvey, J. N., Vanderbrei, R. J., and Zenios, S. A., "Robust optimization of large scale systems," Operations Research, Vol. 2, No. 43, 1995, pp. 264-280.

${ }^{3}$ Gustafson, S. A., Semi-infinite programming and applications, Springer, Berlin, 1981.

${ }^{4}$ Howe, M. A., Rustem, B., and Selby, M. J. P., "Multi-period minimax hedging strategies," European Journal of Operations Research, Vol. 1, No. 93, 1996, pp. 185-204.

24 of 25

American Institute of Aeronautics and Astronautics 
${ }^{5}$ Rustem, B. and Nguyen, Q., "An algorithm for the inequality constrained minimax problem," SIAM Journal of Optimization, Vol. 8, No. 1, 1998, pp. 265-283.

${ }^{6}$ Crespo, L. G., Giesy, D. P., and Kenny, S. P., "Strict Constraint Feasibility in Analysis and Design of Uncertain Systems," 11th AIAA/ISSMO Multidisciplinary Analysis and Optimization Conference, Portsmouth, VA USA, September 2006, pp. 1-28, AIAA-2006-7035.

${ }^{7}$ Crespo, L. G., Giesy, D. P., and Kenny, S. P., "Hard Constraints in Optimization under Uncertainty," AIAA Journal, 2007, (in review).

${ }^{8}$ Acevedo, J. and Pistikopoulos, E. N., "Stochastic Optimization Based Algorithms for Process Synthesis Under Uncertainty," Comp. Chem. Engng, Vol. 22, No. 1, 1998, pp. 647-671.

${ }^{9}$ Samsatli, N., Papageorgiou, L., and Shah, N., "Robustness metrics for Dynamic Optimization Models under Parametric Uncertainty," Process Systems Engineering, Vol. 44, No. 9, 1998, pp. 1993-2006.

${ }^{10}$ Royset, J., Kiureghian, A. D., and Polak, E., "Reliability-based optimal structural design by the decoupling approach," Reliability Engineering and System Safety, Vol. 73, 2001, pp. 213-221.

${ }^{11}$ Crespo, L. G. and Kenny, S. P., "Reliability-based control design for uncertain systems," AIAA Journal of Guidance, Control, and Dynamics, Vol. 28, No. 4, 2005.

${ }^{12}$ Kall, P. and Wallace, S., Stochastic Programming, Wiley, New York, 1994.

${ }^{13}$ Rackwitz, R., "Reliability analysis, a review and some perspectives," Structural Safety, Vol. 23, 2001, pp. 365-395.

${ }^{14}$ Crespo, L. G., Giesy, D. P., and Kenny, S. P., "Hard and Soft Constraints in Reliability-based Optimization," European Safety and Reliability Conference ESREL 2006, Estoril, Portugal, 18-22 September 2006.

${ }^{15}$ Crespo, L. G., Giesy, D. P., and Kenny, S. P., "Reliability-based Analysis and Design via Failure Domain Bounding," Structural Safety, 2007, (in review).

${ }^{16}$ A.Mills, R. and Bryson, A. E., "Parameter-Robust control design using the minimax method," AIAA Journal of Guidance, Control and Dynamics, Vol. 15, No. 5, 1992, pp. 1068-1075.

${ }^{17}$ Bryson, A. E. and A.Mills, R., "Linear-Quadratic-Gaussian Controllers with Specified Parameter Robustness," AIAA Journal of Guidance, Control and Dynamics, Vol. 21, No. 1, 1998, pp. 11-18.

25 of 25

American Institute of Aeronautics and Astronautics 